\title{
Engineered protein A ligands, derived from a histidine-scanning library, facilitate the affinity purification of lgG under mild acidic conditions
}

\author{
Masayuki Tsukamoto ${ }^{1,3}$, Hideki Watanabe ${ }^{2}$, Ayako Ooishi ${ }^{2}$ and Shinya Honda 1,2,3*
}

\begin{abstract}
Background: In antibody purification processes, the acidic buffer commonly used to elute the bound antibodies during conventional affinity chromatograph, can damage the antibody. Herein we describe the development of several types of affinity ligands which enable the purification of antibodies under much milder conditions.

Results: Staphylococcal protein A variants were engineered by using both structure-based design and combinatorial screening methods. The frequency of amino acid residue substitutions was statistically analyzed using the sequences isolated from a histidine-scanning library screening. The positions where the frequency of occurrence of a histidine residue was more than $70 \%$ were thought to be effective histidine-mutation sites. Consequently, we identified PAB variants with a D36H mutation whose binding of lgG was highly sensitive to $\mathrm{pH}$ change.

Conclusion: The affinity column elution chromatograms demonstrated that antibodies could be eluted at a higher $\mathrm{pH}$ $\left(\Delta p H^{* *} \geqq 2.0\right)$ than ever reported $(\Delta p H=1.4)$ when the Staphylococcal protein A variants developed in this study were used as affinity ligands. The interactions between Staphylococcal protein A and lgG-Fab were shown to be important for the behavior of IgG bound on a SpA affinity column, and alterations in the affinity of the ligands for lgG-Fab clearly affected the conditions for eluting the bound lgG. Thus, a histidine-scanning library combined with a structure-based design was shown to be effective in engineering novel pH-sensitive proteins.
\end{abstract}

Keywords: Antibody, Affinity purification, Staphylococcal protein A, Histidine-scanning Library, Combinatorial screening

\section{Background}

Monoclonal antibodies are currently one of the most important applications in biotechnology and as a promising class of drugs in the biopharmaceutical industry $[1,2]$. Affinity chromatography is commonly used for antibody purification on both the laboratory and industrial scale because it provides easy, fast and selective purification [3]. However, conditions for efficiently eluting monoclonal antibodies from affinity columns require improvement. Conventional affinity chromatography for antibodies uses an acidic buffer solution for elution, which would cause antibodies to denature and/or to form aggregates, leading to immunogenicity and other adverse reactions $[4,5]$. To

\footnotetext{
*Correspondence: s.honda@aist.go.jp

'Department of Medical Genome Sciences, Graduate School of Frontier Sciences, The University of Tokyo, Kashiwa, Japan

${ }^{2}$ Biomedical Research Institute National Institute of Advanced Industrial Science and Technology (AIST), Higashi, Tsukuba, Ibaraki 305-8566, Japan Full list of author information is available at the end of the article
}

solve these problems, several types of affinity ligands have been developed for the elution of antibodies under milder conditions [6-11].

The affinity ligands commonly used for antibody purification are Staphylococcal protein A (SpA) and Streptococcal protein $\mathrm{G}(\mathrm{SpG})$. Optimizing the $\mathrm{pH}$-sensitive interactions of these affinity ligands with the target antibodies could improve elution conditions and guarantee the quality of purified antibody. A number of studies using various strategies [7,9-19] to modulate the $\mathrm{pH}$ sensitivity of protein-protein interactions have been carried out. Most of $\mathrm{pH}$-sensitive ${ }^{*}$ proteins in these studies were produced by introducing histidine-mutations based on structural designs, such as that by Brown et al. [7] on engineered $\mathrm{pH}$-sensitive SpA B-domain (PAB) variants. In previous studies, we developed rational strategies for histidine-mutations to produce $\mathrm{pH}$-sensitive IgG-binding SpG [9] and SpA [11].

\section{Biomed Central}


To obtain $\mathrm{pH}$-sensitive proteins, it is important to explore the most effective positions for histidine-substitution. Gera et al. [10] performed a histidine-scanning mutagenesis study where a number of Sso7d protein variants with individual single histidine-substitutions were systematically examined; they identified a $\mathrm{pH}$-sensitive mutant containing a single histidine substitution. In addition to these structurebased designs, combinatorial screening is also a promising approach. Murtaugh et al. [19] reported the engineering of $\mathrm{pH}$-sensitive proteins using a histidine-scanning library of anti-RNase A single domain $\mathrm{VHH}$ antibodies, and identified $\mathrm{pH}$-sensitive antibodies with multiple histidine substitutions that retained near wild-type affinity but with high sensitivity to $\mathrm{pH}$ change for elution.

In this paper, we describe the design and generation of $\mathrm{pH}$-sensitive $\mathrm{PAB}$ variants by introducing a histidinemutation using both structure-based design and combinatorial screening. First, a histidine-scanning library for PAB was created based on structural information. The effective histidine-mutation positions were evaluated from statistical analysis of the frequency of occurrence of amino acids in the sequences isolated using a T7 phage display screening. We also investigated the $\mathrm{pH}$ value at which IgG peaks eluted from PAB variant-immobilized affinity columns. To better understand the effects of histidinemutation at molecular level, we evaluated the structural stability of each histidine-substituted PAB variant and its binding affinity to IgG by Surface Plasmon Resonance (SPR) analysis.

\section{Results and discussion}

\section{Generation of histidine-scanning library}

To design the histidine-scanning library, we focused on the IgG-Fc binding interface of PAB for the mutation positions to be randomized. Our previous study [9] revealed that positions near positively charged residues on a target protein were effective sites for histidine-mutation. First, positively charged residues (histidine, lysine and arginine) on IgG1-Fc under acidic conditions were searched using the structure of PAB complexed with IgG1-Fc [pdb: 1FC2] [20]. Distances from the positively charged residues in IgG1-Fc to all the PAB residues were calculated (Table 1). Next, the solvent exposure of each residue in PAB was calculated in order to select the residues on the protein surface using the subunit structure of PAB in the complex (Table 1).

Several residues important in the interaction between PAB and IgG1-Fc have been previously identified by mutational experiments [7,11,21-24] and by molecular simulation calculations $[25,26]$. However, we did not take these positions into particular consideration for our library design in order to test a wide range of mutation positions in PAB.

The theoretical molecular diversity of a histidinescanning library can be controlled by the number of mutation positions. In order to restrict the library to fit within the size range of $\mathrm{T} 7$ phage-display screening, we determined the maximum number of mutation positions. The seventeen mutation positions were finally selected (Table 1).

\section{Screening of histidine-scanning library}

The histidine-scanning library was screened using a T7 phage-display method. The initial library size $\left(5.4 \times 10^{7}\right.$ pfu) of T7 phages estimated by plaque assays was larger than the theoretical value $\left(2.5 \times 10^{7} \mathrm{pfu}\right)$ of the objective library, so a highly diverse library was produced.

After each round of selection, the T7 phages were cloned and several dozen of the sequences were determined. The ratios of unique sequences out of the sequences determined after each round were as follows (the number of unique sequences/the number of total sequences determined): $100 \%$

Table 1 Mutation positions for histidine-scanning library

\begin{tabular}{|c|c|c|c|c|c|c|c|c|c|c|c|c|c|c|c|c|c|}
\hline Number & 5 & 6 & 9 & 10 & 11 & 13 & 14 & 15 & 17 & 24 & 25 & 27 & 28 & 31 & 32 & 35 & 36 \\
\hline $\begin{array}{c}\text { Aminoacid residue } \\
\text { of wild type }\end{array}$ & $F$ & $\mathrm{~N}$ & Q & Q & $N$ & F & Y & $E$ & L & $E$ & $E$ & $\mathrm{R}$ & $\mathrm{N}$ & I & Q & K & $D$ \\
\hline Distance $(\AA)$ & 5.7 & 11 & 6.4 & 4.2 & 6.5 & 3.8 & 3.4 & 7.9 & 4.0 & 8.4 & 8.7 & 8.6 & 6.9 & 5.8 & 8.1 & 4.5 & 9.8 \\
\hline fSASA (\%) & 78 & 94 & 36 & 47 & 64 & 40 & 63 & 50 & 46 & 78 & 96 & 23 & 71 & 17 & 60 & 64 & 68 \\
\hline $\begin{array}{l}\text { Mixed codon used in } \\
\text { the library }\end{array}$ & YWT & MAY & CAW & CAW & MAY & YWT & YAT & SAW & CWK & SAW & SAW & CRY & MAY & MWT & CAW & MAW & SAT \\
\hline \multirow{3}{*}{$\begin{array}{c}\text { Substituted amino acids in } \\
\text { the library }\end{array}$} & $\mathrm{H}$ & & & & & $\mathrm{H}$ & & $\mathrm{H}$ & $\mathrm{H}$ & $\mathrm{H}$ & $\mathrm{H}$ & & & $\mathrm{H}$ & & $\mathrm{H}$ & \\
\hline & Y & $\mathrm{H}$ & $\mathrm{H}$ & $\mathrm{H}$ & $\mathrm{H}$ & Y & $\mathrm{H}$ & D & Q & $D$ & $D$ & $\mathrm{H}$ & $H$ & $\mathrm{~N}$ & $\mathrm{H}$ & $\mathrm{N}$ & $\mathrm{H}$ \\
\hline & $\mathrm{L}$ & & & & & $L$ & & Q & & Q & Q & & & $\mathrm{L}$ & & Q & \\
\hline
\end{tabular}

Distance in the table indicates the length between the PAB residue and the nearest positively charged residue in IgG-Fc. fSASA indicates the extent of solvent exposure of the side chain in wild-type amino acid residues of PAB. The seventeen mutation positions finally selected were classified into two groups: (1) the PAB residues were located within $9 \AA$ from the positively charged lgG-Fc residues and (2) the PAB residues were located between 9 and $11 \AA$ from the lgG-Fc residues, and with a fractional solvent-accessible surface area (fSASA) ((fSASA) $=(\mathrm{SASA})$ (native)/SASA (denatured)) value of more than $68 \%$. The following abbreviations are used for mixed bases: $R=(A$ or $G), Y=(C$ or $T), M=(A$ or $C), K=(G$ or $T), S=(G$ or $C)$ and $W=(A$ or $T)$. Substituted amino acid residues in the library indicate non-wild-type residues encoded by a mixed codon. 
(the initial library: 84/84), 100\% (after the 1st round: 50/50), 97.5\% (after the 2nd round: 78/84), 51.7\% (after the 3rd round: $30 / 58$ ), $25.0 \%$ (after the 4th round: 20/88), and $19.3 \%$ (after the 5th round: 18/93). Sixty one out of 93 clones examined after the final round had identical sequences (Additional file 1: Table S2). It should be mentioned that the frequency of occurrence of the wild-type or histidine residue at each mutation position fluctuated (Additional file 2: Figure S1, Additional file 3: Figure S2 and Additional file 4: Figure S3).

\section{Frequency of occurrence of amino acid residues of isolated $\mathrm{PAB}$ variants}

The frequency of occurrenc of the amino acids was calculated from 18 unique sequences determined from the final round (Figure 1). The positions at which the frequency of the histidine residue was less than 20\% were: F13, Y14, L17, E24 and I31, which were previously shown to be essential for the interaction with IgG-Fc [6,7,21-26]. For example, Brown et al. [7] used ELISA to show that the binding affinity of rabbit IgG with a PAB mutant (F13H) at $\mathrm{pH} 8.0$ was more than 100 -fold weaker than with wildtype PAB.

The positions at which the frequency of occurrence of a histidine residue was more than $70 \%$ were expected to be effective histidine-mutation sites. They were: Q9, Q10, R27, K35 and D36. The frequency of a histidine residue at D36 was the highest, although D36 has been little investigated in previous studies [7,21-26]. Hence, a single histidine-substituted $(\mathrm{D} 36 \mathrm{H}) \mathrm{PAB}$ variant and four double histidine-substituted $\mathrm{PAB}$ ones containing the D36H mutation and either the Q9H, Q10H, R27H or $\mathrm{K} 35 \mathrm{H}$ mutation were produced.

\section{Elution profile of bound IgG from the PAB variant columns}

The affinity chromatograms with the PAB variants described above indicated that the $\mathrm{pH}$ values at which IgG eluted from all the $\mathrm{PAB}$ variant columns were higher than the elution pH from the PAB01 (Wild-type (WT)) column (Table 2, Additional file 5: Figure S4). First, the $\mathrm{pH}$ sensitivity of PAB02 (D36H) was clearly higher. For the double substitution $\mathrm{PAB}$ variants, the $\mathrm{pH}$ values of the IgG elution peaks from the PAB03 $(\mathrm{Q} 9 \mathrm{H}, \mathrm{D} 36 \mathrm{H})$ and PAB04 (Q10H, D36H) columns were significantly higher than that of the PAB02 (D36H) column. On the other hand, the $\mathrm{pH}$ required to elute IgG from the PAB05 (R27H, $\mathrm{D} 36 \mathrm{H})$ and PAB06 (K35H, D36H) column was similar to that of the PAB02 column. Therefore, the Q9, Q10 and D36 histidine-mutations showed much better $\mathrm{pH}$ sensitivity. The $\triangle p H$ of the IgG elution peaks from the PAB03 and PAB04 columns was more than ever reported (Table 2, Additional file 6: Table S3).

Next, we evaluated the effects of combinations of the histidine-mutations. The $\mathrm{pH}$ values for eluting IgG from a single histidine-substituted PAB variant (PAB07 $(\mathrm{Q} 9 \mathrm{H})$ or PAB08 $(\mathrm{Q} 10 \mathrm{H})$ ) column was slightly higher than that required with $\mathrm{PAB} 01$. Thus, the large effects of $\mathrm{Q} 9 \mathrm{H}$ and $\mathrm{Q} 10 \mathrm{H}$ on the $\mathrm{pH}$ sensitivity of the PAB03 and PAB04 columns were likely due to the combination effect with D36H.

\section{Structural stability of PAB variants}

We attempted to evaluate the effect of introducing a histidine-mutation on the structure and thermal stability of PAB variants. The circular dichroism $(C D)$ spectra of all $\mathrm{PAB}$ variants at room temperature were similar to

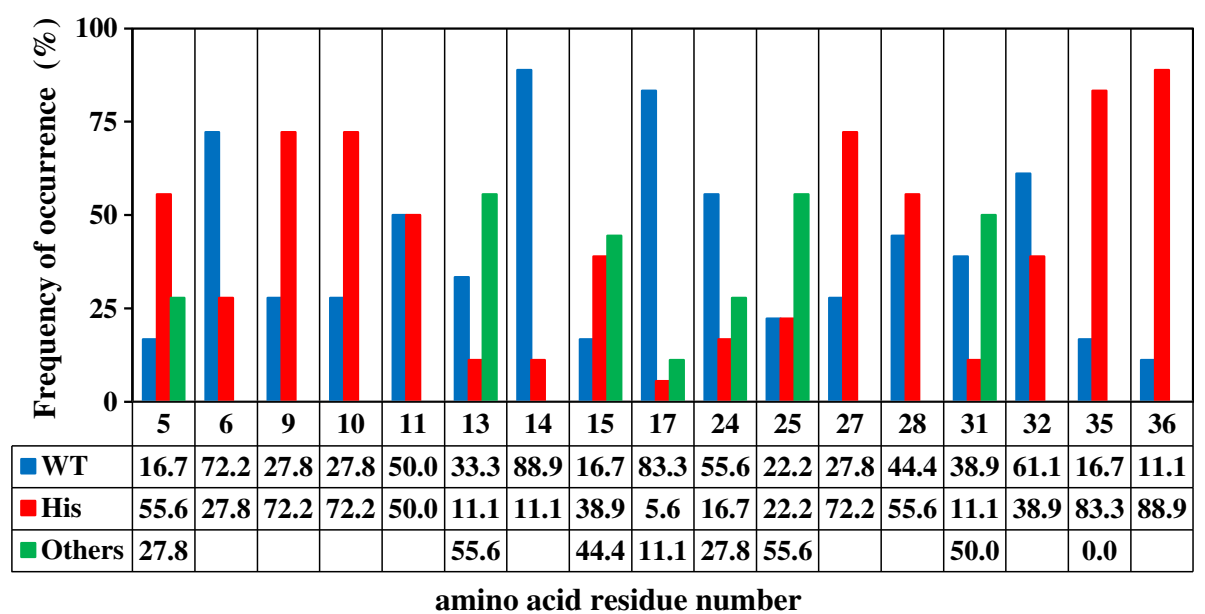

Figure 1 Frequency of occurrence of amino acid residues after the final round. The frequency of occurrence of amino acid residues was calculated from 18 unique determined sequences of PAB variants after the final round. The ordinate indicates the percentage of the frequency of occurrence. The abscissa indicates the amino acid residue number. WT, wild-type residue: His, histidine residue: Others, non-wild-type and non-histidine residues. The number in the lower three rows indicate percentage. 
Table 2 The pH values of the IgG elution peaks and the thermal stability of PAB variants

\begin{tabular}{|c|c|c|c|c|}
\hline Protein & $\begin{array}{c}\text { Elution Peak } \\
\mathrm{pH}\end{array}$ & $\Delta p H$ & $\begin{array}{c}\text { Stability } \\
T_{\mathrm{m}}(\mathrm{K})\end{array}$ & $\Delta T_{\mathrm{m}}$ \\
\hline PAB01 (Wild Type) & 3.5 & - & 346.4 & - \\
\hline PAB02 (D36H) & 4.6 & 1.1 & 345.8 & -0.6 \\
\hline PAB03 (Q09H, D36H) & 5.5 & 2.0 & 343.7 & -2.7 \\
\hline PAB04 (Q10H, D36H) & 6.8 & 3.3 & 343.1 & -3.3 \\
\hline PAB05 (R27H, D36H) & 4.7 & 1.2 & 316.2 & -30.2 \\
\hline PAB06 (K35H, D36H) & 4.4 & 0.9 & 342.5 & -3.9 \\
\hline PAB07 (Q09H) & 3.9 & 0.4 & 344.9 & -1.5 \\
\hline PAB08 (Q10H) & 3.7 & 0.2 & 343.6 & -2.8 \\
\hline PAB10 (Q32H) & 4.2 & 0.7 & 341.4 & -5.0 \\
\hline
\end{tabular}

Affinity columns were prepared using the PAB variants. The captured IgG on each column was eluted with a decreasing $\mathrm{pH}$ gradient. The $\mathrm{pH}$ value in the table indicates the peak position of the eluted IgG on the affinity chromatography. The midpoint of the transition during thermal denaturation $\left(T_{\mathrm{m}}\right)$ was determined from circular dichroism melting measurements. $\Delta p H=$ (the $\mathrm{pH}$ value at which $\mathrm{lgG}$ eluted from mutant column) - (the $\mathrm{pH}$ value at which IgG eluted from wild-type column).

$\Delta T_{\mathrm{m}}=$ (the thermal stability of PAB mutant expressed as $T_{\mathrm{m}}$ ) - (that of the thermal stability of $\mathrm{PAB}$ wild-type, $\mathrm{PAB} 01$ ).

that of PAB01 (WT). The midpoint of the transition during thermal denaturation $\left(T_{\mathrm{m}}\right)$ was determined from CD melting measurements (Table 2, Additional file 7: Figure S5). The $\mathrm{pH}$-sensitive $\mathrm{PAB}$ variants with effective histidine-mutations $(\mathrm{Q} 9 \mathrm{H}, \mathrm{Q} 10 \mathrm{H}$ and $\mathrm{D} 36 \mathrm{H})$ showed a slight decrease in thermal stability. An increase in the number of histidine-mutations led to a decrease in the $T_{\mathrm{m}}$ value. Thus, histidine-mutations apparently have additive effects on thermal stability. In summary, however, the decrease in thermal stability due to histidine-mutations was rather limited and would not prevent utilization of these mutants as affinity ligands.

\section{Binding assay of PAB variants Interaction under neutral conditions}

We examined the binding affinity of IgG for each of the four single histidine-substituted $P A B$ variants under neutral conditions $(\mathrm{pH}=7.4)$ (Table 3). Of these, PAB08 $(\mathrm{Q} 10 \mathrm{H})$ showed the weakest binding affinity, about 10-fold weaker than that of PAB01 (WT). The binding affinities of IgG for the double histidine-substituted PAB variants containing $\mathrm{D} 36 \mathrm{H}$ were lower than those of their single substitution $\mathrm{PAB}$ variant counterparts. PAB03 (Q9H, D36H) was exhibited 56-fold weaker binding affinity than PAB01. Thus, the IgG binding of PAB variant columns in this study was decreased (Additional file 8: Figure S6). Still, except for PAB04, the interaction between IgG and each of the PAB variants with single or double histidine substitutions would be strong enough to retain IgG on an affinity column using these variants as ligands.
Table 3 Binding affinity of IgG for PAB variants

\begin{tabular}{lccccc}
\hline \multirow{2}{*}{ Protein } & \multicolumn{2}{c}{$\mathbf{p H}=\mathbf{7 . 4}$} & & \multicolumn{2}{c}{$\mathbf{p H}=\mathbf{5 . 0}$} \\
\cline { 2 - 3 } \cline { 6 - 7 } & $\begin{array}{c}\boldsymbol{K}_{\mathrm{D}}\left(\times \mathbf{1 0}^{-\mathbf{9}}\right) \\
\left(\mathbf{M}^{-1}\right)\end{array}$ & $\boldsymbol{K}_{\mathrm{D}}$ ratio & & $\begin{array}{c}\boldsymbol{K}_{\mathrm{D}}\left(\times \mathbf{1 0}^{-\mathbf{9}}\right) \\
\left(\mathbf{M}^{-1}\right)\end{array}$ & $\boldsymbol{K}_{\mathrm{D}}$ ratio \\
\hline PAB01 (WT) & $5.1 \pm 3.2$ & - & & $7.6 \pm 4.3$ & - \\
PAB02 (D36H) & $33.6 \pm 19.2$ & 6.6 & & $832 \pm 443$ & 110 \\
PAB03 (Q9H, D36H) & $288 \pm 146$ & 56 & & $\mathrm{ND}$ & - \\
PAB04 (Q10H, D36H) & $6830 \pm 3600$ & 1300 & & $\mathrm{ND}$ & - \\
PAB05 (R27H, D36H) & 80.7 & 16 & & 2090 & 270 \\
PAB06 (K35H, D36H) & $550 \pm 143$ & 110 & & 3860 & 510 \\
PAB07 (Q9H) & $13.9 \pm 6.3$ & 2.7 & & 30.6 & 4 \\
PAB08 (Q10H) & $50.8 \pm 20.5$ & 10 & & 951 & 120 \\
PAB09 (R27H) & $5.6 \pm 1.3$ & 1.1 & & 7.0 & 0.92 \\
PAB10 (Q32H) & $2.8 \pm 1.3$ & 0.55 & & 96.8 & 13 \\
\hline
\end{tabular}

The binding assay was performed under neutral $(\mathrm{pH}=7.4)$ and acidic $(\mathrm{pH}=5.0)$ conditions using SPR. The IgG was immobilized on the surface of a CM5 sensor chip (GE Healthcare).

$K_{\mathrm{D}}$, equilibrium dissociation constant: $K_{\mathrm{D}}$ ratio, $\left(K_{\mathrm{D}}\right.$ of histidine-substituted $\mathrm{PAB}$ variant $) /\left(K_{D}\right.$ of Wild-type $\left.P A B\right): N D$, not determined.

\section{Interaction under acidic conditions}

The binding affinity of IgG for PAB01 under the acidic condition $(\mathrm{pH}=5.0)$ (Table 3) was about 1.5 -fold weaker than at neutral $\mathrm{pH}$, still quite strong. PAB07 $(\mathrm{Q} 9 \mathrm{H})$ and PAB09 (R27H) also retained strong affinity. Brown et al. [7] showed that the $\mathrm{K} 35 \mathrm{H} \mathrm{PAB}$ variant exhibited strong affinity under both neutral $(\mathrm{pH}=8.0)$ and acidic $(\mathrm{pH}=5.0)$ conditions.

On the other hand, the binding affinity of IgG for PAB02 $(\mathrm{D} 36 \mathrm{H})$ and PAB08 $(\mathrm{Q} 10 \mathrm{H})$ was more than 100-fold weaker than that for PAB01. The binding affinities for PAB03 (Q9H, D36H) and PAB04 (Q10H, D36H) were too weak to be determined under the acidic condition. Thus, histidine-mutations at Q9, Q10 and D36, as well as the double-mutations, Q9H plus D36H and Q10H plus D36H, appeared to have favorable $\mathrm{pH}$-sensitivity. It should also be noted that histidine-mutation at D36 improved the $\mathrm{pH}$ sensitivity of all the four histidine-mutations under both neutral and acidic conditions.

\section{The effects of histidine-mutations}

The observed effects of the histidine-mutations could be explained based on crystal structure and biophysical data. Q9 and Q10 of PAB probably contribute to the interaction with IgG-Fc. The binding affinity of PAB08 (Q10H) under the neutral condition was about 10 -fold weaker than that of PAB01 (WT). Five residues in IgG-Fc are found within $4 \AA$ of Q10 of PAB. Therefore, the steric hindrance generated by the $\mathrm{Q} 10 \mathrm{H}$ mutation likely affects the interaction with IgG-Fc. IgG elution from the affinity columns and SPR measurement results under the acidic condition demonstrated that the effects of histidine-mutation at Q10 are more significant than mutation at Q9. Because the positively 
charged residue (His435) in IgG-Fc resided near Q10 of $\operatorname{PAB}(<4.2 \AA)$, electrostatic repulsion would increase when the $\mathrm{pH}$ value changes from neutral to acidic.

As described below, however, the decrease in binding affinity due to the D36H mutation (PAB02) was unlikely to be affected by modulation of the interaction of the IgG-Fc binding interface of PAB.

\section{Effect of the D36H mutation}

Ile253 of IgG-Fc resides $6.89 \AA$ from D36 of PAB in the PAB-IgG1-Fc complex [20]. Since this is not a short distance, the effects of the histidine-mutation at D36 obtained from the screening experiment are difficult to explain if the effect were caused by modulation of the interaction between PAB and IgG-Fc. In fact, it was previously observed that SpA binds not only to IgG-Fc but also to IgG-Fab [27-30]. We focused on the IgG-Fab binding interface of PAB.

We evaluated the binding affinity of IgG-Fc and IgGFab for PAB01 (WT) and PAB02 (D36H) (Tables 3 and 4) using SPR. The interaction between PAB02 and IgG-Fc was found to be similar to that of PAB01 under both neutral and acidic conditions. On the other hand, the binding affinity of PAB02 for IgG-Fab under the neutral condition was 2.3-fold weaker than that of PAB01, and the binding affinity of PAB02 under the acidic condition was too weak to be determined. These results suggested that the interaction of IgG-Fab with PAB would significantly contribute to the effects of histidine-mutation at D36.

Next, we analyzed the effects of D36H using the structure of SpA D-domain complexed with IgG-Fab [30]. D36 is conserved in all the SpA domains (A, B, C, D and E). The crystal structure indicates that six residues in IgG-Fab are within $4 \AA$ of D36 in PAB. Therefore, the steric hindrance due to the histidine-mutation of D36 would affect the interaction with IgG-Fab under neutral conditions.

Table 4 Binding affinity of IgG-Fc (upper rows) or IgG-Fab (lower rows) for PAB01 (WT) and PAB02 (D36H)

\begin{tabular}{|c|c|c|c|c|}
\hline \multirow[t]{2}{*}{ Protein } & \multicolumn{2}{|c|}{$\mathrm{pH}=7.4$} & \multicolumn{2}{|c|}{$\mathrm{pH}=5.0$} \\
\hline & $\begin{array}{c}K_{\mathrm{D}}\left(\times 10^{-9}\right) \\
\left(\mathrm{M}^{-1}\right)\end{array}$ & $K_{\mathrm{D}}$ ratio & $\begin{array}{c}K_{\mathrm{D}}\left(\times 10^{-9}\right) \\
\left(\mathrm{M}^{-1}\right)\end{array}$ & $K_{\mathrm{D}}$ ratio \\
\hline PAB01 (WT) & 12.8 & - & 1080 & - \\
\hline \multirow[t]{2}{*}{ PAB02 (D36H) } & 15.5 & 1.2 & 1350 & 1.3 \\
\hline & \multicolumn{2}{|c|}{$\mathrm{pH}=7.4$} & \multicolumn{2}{|c|}{$\mathrm{pH}=5.0$} \\
\hline Protein & $\begin{array}{c}K_{\mathrm{D}}\left(\times 10^{-5}\right) \\
\left(\mathrm{M}^{-1}\right)\end{array}$ & $K_{\mathrm{D}}$ ratio & $\begin{array}{c}K_{\mathrm{D}}\left(\times 10^{-5}\right) \\
\left(\mathrm{M}^{-1}\right)\end{array}$ & $K_{\mathrm{D}}$ ratio \\
\hline PAB01 (WT) & 2.9 & - & ND & - \\
\hline PAB02 (D36H) & 6.7 & 2.3 & ND & - \\
\hline
\end{tabular}

The binding assay was performed under neutral $(\mathrm{pH}=7.4)$ and acidic $(\mathrm{pH}=5.0)$ conditions using SPR. IgG-Fc or IgG-Fab was immobilized on the surface of a CM5 sensor chip (GE Healthcare).

$K_{\mathrm{D}}$, equilibrium dissociation constant: $K_{\mathrm{D}}$ ratio, $\left(K_{\mathrm{D}}\right.$ of histidine-substituted $\mathrm{PAB}$ variant $) /\left(K_{D}\right.$ of Wild-type $\mathrm{PAB}$ or $\left.\mathrm{PAZ}\right)$ : ND, not determined.
Because a positively charged residue (Arg2519) in IgG-Fab is present near D36 in PAB $(<3.0 \AA)$, electrostatic repulsion under acidic conditions should be stronger than under neutral conditions.

We also focused on Arg2519 in IgG-Fab as it would be involved in the effects of histidine-mutation of D36. Q32 of PAB is located within $4 \AA$ of Arg2519 of IgGFab. The PAB Q32H variant (PAB10) was produced, and the $\mathrm{pH}$ value of the IgG elution peaks from the affinity column with PAB10 was found to be significantly higher than those values for PAB01 (WT), PAB07 (Q9H) and PAB08 $(\mathrm{Q} 10 \mathrm{H})$ columns. This suggested that the introduction of histidine-mutations into the IgG-Fab binding interface of PAB was as effective as introducing them into the IgG-Fc binding interface. In addition, the majority of amino acid residues corresponding to Arg2519 of the top 10 therapeutic antibodies are positively charged ones (Additional file 9: Table S4). Therefore, the PAB affinity ligands with $\mathrm{D} 36 \mathrm{H}$ mutation should be effective for therapeutic antibody purification.

Although the binding affinity of IgG-Fab for PAB01 (WT) was more than 1000-fold weaker than that of IgGFc (Table 4), a possible reason for the remarkable effects of the D36H mutation would be a change in avidity. IgG has four SpA binding sites: two are strong binding sites in the IgG-Fc region and two are weak binding sites in the IgG-Fab region. On an affinity column, since a plurality of interactions for these four sites would bind the IgG molecule, the cooperative effect of interactions between SpA and IgG would be strong. Since the D36H mutation would result in just 2 binding sites instead of 4 in the interaction with IgG, the effect of avidity would likely decrease significantly, thus promoting dissociation under moderately acidic conditions.

\section{Elution profile of bound IgG from $4 \times \mathrm{PAZ}$ variant columns} An affinity column of tandem SpA $(4 \times$ PAZ) consisting of four SpA Z-domains was fabricated using $\mathrm{pH}$-sensitive double substituted $\mathrm{PAB}$ variant (PAB03) and its performance was compared with commercially available affinity columns (Figure 2).

IgG was eluted with a stepwise $\mathrm{pH}$ decrease. The relative amounts of IgG eluted under mild acidic conditions ( $\mathrm{pH}=5 \sim 6$ ) was $67 \%$ at $\mathrm{pH}=6.0,82 \%$ at $\mathrm{pH}=5.5$ and $90 \%$ at $\mathrm{pH}=5.0$. In contrast, IgG bound to the $4 \times$ PAZ01 column or to a commercially available SpA column (rProtein A) (GE Healthcare) did not elute at $\mathrm{pH}=5.0$. Although a part of IgG injected onto the $4 \times$ PAZ03 column seemed to flow through, more than the case of the $4 \times$ PAZ01 column, with $\mathrm{pH} 7.5$ binding buffer, the amounts of IgG leaking out were very little, if any, with $\mathrm{pH} 9.0$ binding buffer (Additional file 10: Figure S7). Thus, the $4 \times$ PAZ03 (Q9H, $\mathrm{D} 36 \mathrm{H})$ column would allow the retention of IgG by optimizing the affinity column condition and the elution from 


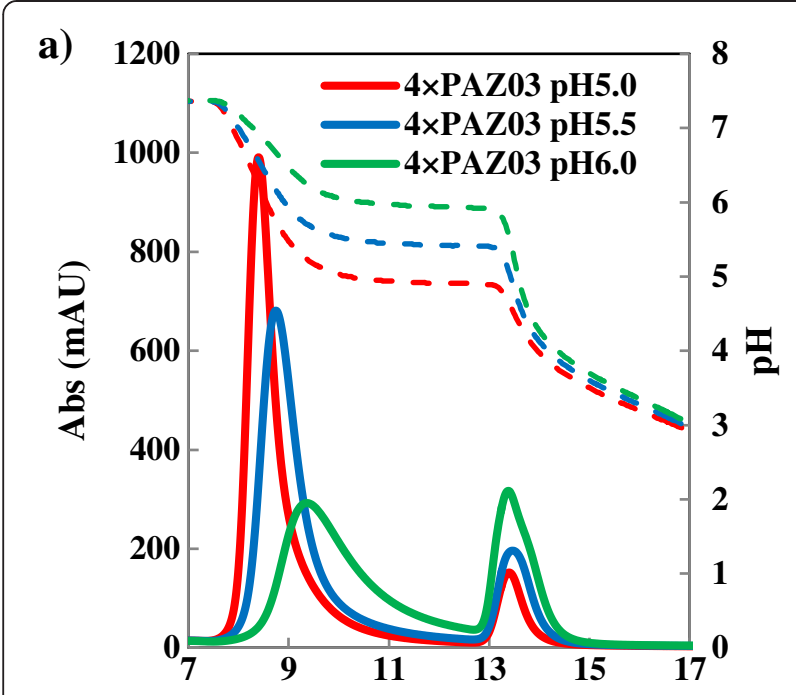

b) $1600 \square 8$

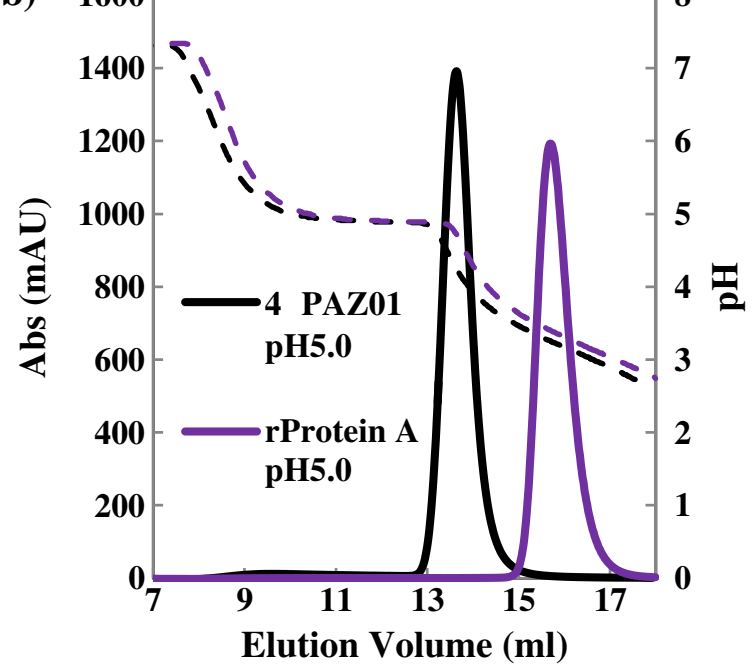

Figure 2 Elution profile of IgG on affinity columns with immobilized $4 \times$ PAZ variants. (a) Elution profile of $\mathrm{lgG}$ from affinity columns prepared with immobilized $4 \times$ PAZ03. IgG was eluted with $\mathrm{pH} 5.0$, pH 5.5 or pH 6.0 buffer, (b) Elution profile of $\mathrm{lgG}$ from affinity columns prepared with immobilized $4 \times$ PAZ01 or rProteinA (GE Healthcare). Elution was performed using pH 5.0 buffer. Affinity columns were prepared using the $4 \times$ PAZ variants. The captured IgG on the column was eluted using a buffer with the $\mathrm{pH}$ indicated in the Figure. Afterwards, the residual lgG was eluted with a decreasing $\mathrm{pH}$ gradient. The ordinate on the right indicates the $\mathrm{pH}$ of the solution. The ordinate on the left indicates the absorbance at $280 \mathrm{~nm}$ (mAU: milliabsorbance units). The abscissa indicates the elution volume. The solid and dashed lines show the elution patterns of IgG on $4 \times$ PAZ variants or rProteinA (GE Healthcare) columns, as shown in the Figure, and the $\mathrm{pH}$ value of the elution buffer, respectively.

the column under much milder $\mathrm{pH}$ conditions than previously reported $[7,8,11]$.

\section{Conclusion}

We have shown that a histidine-scanning library combined with a structure-based design was effective in engineering novel $\mathrm{pH}$-sensitive proteins. The positions at which histidine residues occurred with high frequency should be suitable sites for introducing histidine-mutations. Large effects of PAB mutations such as $\mathrm{Q} 9 \mathrm{H}$ and $\mathrm{Q} 10 \mathrm{H}$, combined with $\mathrm{D} 36 \mathrm{H}$, were observed. The chromatograms demonstrated that antibodies could be eluted at a higher $\mathrm{pH}$ than ever reported previously when the protein A variants developed in this study were used as affinity ligands. The interactions between SpA and IgG-Fab are apparently important for the behavior of IgG bound on a SpA affinity column, and alterations in the affinity of the ligands for IgG-Fab affected the elution condition for bound IgG. Therefore, not only the interactions with IgG-Fc but also with IgG-Fab should be considered when designing a new affinity ligand for IgG purification.

\section{Materials and methods}

\section{Histidine-scanning library design}

Distances from positively charged residues (histidine, lysine and arginine residues) on IgG1-Fc to all residues of $\mathrm{PAB}$ in the crystal structure of the complex of $\mathrm{PAB}$ bound to IgG1-Fc [pdb: 1FC2] (Table 1) [20] were calculated using the program CONTACT in CCP4 suite [31]. The solvent-accessible surface area (SASA) was calculated for the subunit structure of PAB in the complex. The extent of solvent exposure of all residues of PAB was determined by the equation (Table 1) fSASA (a fractional solvent-accessible surface area) $=$ SASA (native) $/$ SASA (denatured), where a Gly-Xaa-Gly tripeptide model was assumed to adopt the denatured structure. The calculation was performed using surface Racer 3.0 [32].

Gene fragments for constructing a mutated PAB library were prepared with chemically synthesized oligonucleotides (Additional file 11: Table S1), purchased from Rikaken, by the overlap extension method using combinations of these oligonucleotides. At the mutation position of the oligonucleotide, we introduced mixed codons comprising three mixed nucleotides, encoding either the wild-type residue or a histidine residue. However, genes encoding neither the wild-type nor a histidine residue at that position were also produced, since the codon was a random mixture of three nucleotides. Consequently, genes encoding three or four different kinds of residues were obtained for several mutation positions.

The gene fragments thus prepared were treated with restriction enzymes EcoR I and Hind III and linked to the 3 '-terminal side of the g10 gene on the T7 phage genome through ligation reaction $\left(16^{\circ} \mathrm{C}, 16 \mathrm{hr}\right)$ with $\mathrm{T} 7$ phage vector (T7Select1-1b) (Novagen). The linked T7 phage genome was subjected to an in vitro $\mathrm{T} 7$ phage particle packaging reaction $\left(22^{\circ} \mathrm{C}, 2 \mathrm{hr}\right)$ to prepare the initial library.

An E. coli BLT5403 strain cultured in $200 \mathrm{~mL}$ LB medium to O.D. $600=1.0$ was infected with this initial 
library, and a T7 phage library was collected by amplification. About 4 hours after infection, the amplified phages were recovered (T7 phages have bacteriolytic ability and are thus released from the bacterial body) in the supernatant after centrifugation. To the supernatant, 1/6 volume of 50\% polyethylene glycol (PEG, molecular weight: 8000 ) and $1 / 10$ volume of $5 \mathrm{M} \mathrm{NaCl}$ were added, and the mixture was stirred at $4^{\circ} \mathrm{C}$ overnight. The PEG-precipitated phages were partially purified by centrifugation. Then, the phages were lysed by repeated pipetting in TBST buffer $(10 \mathrm{mM}$ Tris- $\mathrm{HCl}(\mathrm{pH}=7.5), 150 \mathrm{mM} \mathrm{NaCl}$, and $0.1 \%$ Tween 20 ) and the resultant suspension was filtered through a $0.22 \mu \mathrm{m}$ filter to obtain the phage library displaying the mutated PAB. This is referred to as the initial phage library.

\section{Phage panning and selection}

Human monoclonal IgG1 antibody (Chugai) was biotinylated using NHS-biotin (Roche) according to the manufacturer's protocol. The biotinylated monoclonal antibody was mixed with streptavidin magnetic beads (Promega) to immobilize the antibodies on the magnetic beads.

For the binding step, the suspension of the phage library $\left(10^{9} \sim 10^{11}\right.$ phage $/ \mathrm{mL}$ titer $)$ displaying the mutated PAB prepared above was added to the biotinylated antibodyimmobilized magnetic beads, and the mixture was shaken at $25^{\circ} \mathrm{C}$ for 1 hour. Afterwards, the beads with bound phages were pulled down by using a magnetic stand (Promega). The beads were washed 10 to 20 times with TBST buffer to remove antibody-unbound phages.

For the elution step, phages specifically bound with the antibodies were recovered by adding a $50 \mathrm{mM}$ sodium acetate solution $(\mathrm{pH}=5.0)$. The eluted phages were subjected to the infection/amplification/PEG-precipitation operation described in the preceding paragraph to prepare the phage library displaying the mutated PAB. This is referred to as the phage library after the first round.

The operation described above, i.e., the screening experiment, was repeated 5 times in total. The eluting solution used in the screening experiment from the second round onwards was $50 \mathrm{mM}$ sodium acetate $(\mathrm{pH}=6.0)$. The selective pressure of screening was changed at each stage by gradually decreasing the amount of biotinylated antibody-immobilized magnetic beads.

\section{Protein preparation}

Expression vectors for single or double histidinesubstituted PAB variants were prepared using a QuickChange $^{\text {tw }}$ site-directed mutagenesis kit (Stratagene). The expression vector for wild-type PAB (pET16-b) was used as the template. The primer sequences (Rikaken) used for this procedure are shown in Additional file 11: Table S1.

The expression vectors for the tandem SpA Z-domain $(4 \times$ PAZ $)$ variants comprised artificial pET16-b vectors containing four sets of the SpA Z-domain gene and the C-terminal cysteine codon and were purchased from Genscript.

E. coli strain BL21 (DE3) was transformed with these plasmid vectors and cultured in $2 \times \mathrm{YT}$ medium containing $100 \mu \mathrm{g} / \mathrm{ml}$ ampicillin to O.D. $600=0.8$ to 1.0 at $37^{\circ} \mathrm{C}$. Recombinant gene expression of $\mathrm{PAB}$ and the $4 \times \mathrm{PAZ}$ variants was induced by the addition of isopropylthio- $\beta$ D-galactopyranoside (IPTG) to a final concentration of $1 \mathrm{mM}$, then the transformants were further cultured at $37^{\circ} \mathrm{C}$ for 2 hours. The bacteria harvested were suspended in TBST buffer and homogenized by ultrasonication. $\mathrm{PAB}$ and $4 \times \mathrm{PAZ}$ variants were purified from the cell lysate by IgG Sepharose 6 Fast Flow chromatography (GE Healthcare). The purity of the sample was checked with Tricine-SDS-PAGE.

\section{Affinity chromatography}

To prepare a $\mathrm{PAB}$ variant-immobilized affinity column, a purified $\mathrm{PAB}$ variant was applied to a HiTrap NHS HP column (GE Healthcare), incubated at room temperature (overnight), and then washed with $0.5 \mathrm{M}$ acetic acid (pH 2.5). Affinity chromatography was performed with an AKTA purifier (GE Healthcare) using the columns as above. Human IgG1 was injected onto the column equilibrated with TBST buffer. The captured IgG was eluted with a decreasing $\mathrm{pH}$ gradient by using $50 \mathrm{mM}$ sodium citrate $(\mathrm{pH}=7.0)$ and $0.5 \mathrm{M}$ acetic acid $(\mathrm{pH}=$ 2.5) (Table 2, Additional file 5: Figure S4). The chromatograms obtained were processed using UNICORN version 4.12 (GE Healthcare).

To prepare a tandem SpA Z-domain $(4 \times \mathrm{PAZ})$ variantimmobilized affinity column, a purified $4 \times$ PAZ variant was chemically coupled to sepharose resin through the sulfhydryl group of the C-terminal cysteine using a SulfoLink Immobilization kit (Pierce). Affinity chromatography was performed as above. The captured IgG was eluted using $50 \mathrm{mM}$ sodium citrate $(\mathrm{pH}=5.0,5.5$ or 6.0$)$. Afterwards, the residual IgG on the $4 \times$ PAZ column was eluted with a decreasing $\mathrm{pH}$ gradient by using $50 \mathrm{mM}$ sodium citrate $(\mathrm{pH}=5.0,5.5$, or 6.0$)$ and $0.5 \mathrm{M}$ acetic acid $(\mathrm{pH}=$ 2.5) (Figure 2).

\section{Stability analysis}

PAB variants were dissolved in $50 \mathrm{mM}$ sodium phosphate $(\mathrm{pH}=7.0)$. Circular dichroism (CD) spectra were obtained on a J-805 spectropolarimeter (JASCO) at various temperatures. CD melting curves were obtained by monitoring the ellipticity at $222 \mathrm{~nm}$ with increasing temperature from 278 to $373 \mathrm{~K}$ at a heating rate of $1.0 \mathrm{~K} / \mathrm{min}$. The 
thermodynamic parameters of proteins for equilibrium unfolding were obtained through a fitting calculation using a two-state transition model [33] on the assumption that heat capacity changes $\left(\Delta C_{p}\right)$ of all proteins are constant and fixed to the value of the wild-type domain $\left(\Delta C_{p}=\right.$ $2.643 \mathrm{~kJ} / \mathrm{mol} \bullet \mathrm{K}$ ) (Table 2). The value of $\Delta C_{p}$ was estimated using the linear relationship between molecular weight and $\Delta C_{p}$ displayed by various proteins [34]. Numerical fitting calculations were carried out using IGOR software (Wavemetrics). Other procedures were the same as described previously [35].

\section{Binding affinity analysis}

Binding analyses of a PAB variant to IgG, IgG-Fc and IgG-Fab were performed using a Biacore T100 (GE Healthcare). Either 4000 resonance units (RU) of human monoclonal IgG1, $1250 \mathrm{RU}$ of the Fab region of human polyclonal antibody (Jackson Immunoresearch), or 1250 RU of the Fc region of human polyclonal antibody (Jackson Immunoresearch) was immobilized on the surface of a CM5 sensor chip (GE Healthcare) using amine coupling chemistry. A binding assay was performed under neutral and acidic conditions as follows: (1) $10 \mathrm{mM}$ HEPES ( $\mathrm{pH}=7.4), 150 \mathrm{mM} \mathrm{NaCl}$ and Tween $20(0.05 \%)$; (2) $10 \mathrm{mM}$ sodium acetate $(\mathrm{pH}=5.0), 150 \mathrm{mM} \mathrm{NaCl}$ and Tween $20(0.05 \%)$. The binding data was fitted to a 1:1 binding model to determine the dissociation constant $\left(K_{\mathrm{D}}\right)$ using Biacore T100 Evaluation software (Tables 3 and 4).

\section{Endnote}

"In this study, ligand proteins with favorable $\mathrm{pH}$ sensitivities are referred to as "pH-sensitive". $* \Delta p H=$ (the $\mathrm{pH}$ value at which IgG eluted from mutant column) - (the $\mathrm{pH}$ value at which IgG eluted from wild-type column).

\section{Additional files}

Additional file 1: Table S2. Eighteen unique sequences of PAB variants after the final round. Amino acid residues at the indicated position number are shown for $P A B_{\text {wild-type }}$ and for 18 unique sequences from the final round, $P A B_{\text {T7phage }}$ 01-18.

Additional file 2: Figure S1. Frequency of occurrence of amino acid residues for each mutation position (5F, 6N, 9Q, 10Q, 11N and 13F). The frequency of occurrence of amino acid residues was calculated from the determined sequences of PAB variants after each round. The title of each section of the Figure, such as Position 5F, indicates the wild-type residue (in this case, F) of the mutation position. The ordinate indicates the percentage of the frequency of occurrence. The abscissa indicates the round number. Wild-type residue (blue), Histidine residue (red), Non-wild-type and non-histidine residues (green or purple).

Additional file 3: Figure S2. Frequency of occurrence of amino acid residues for each mutation position (14Y, 15E, 17L, 24E, 25E and 27R). see the caption in Additional file 2: Figure S1.

Additional file 4: Figure S3. Frequency of occurrence of amino acid residues for each mutation position (28N, 31I, Q32, 35K and 36D). see the caption in Additional file 2: Figure S1.
Additional file 5: Figure S4. Elution profiles of lgG on affinity columns with immobilized PAB variants. Affinity columns were prepared using the PAB variants. The captured lgG on the column was eluted with a decreasing $\mathrm{pH}$ gradient. The ordinate on the right indicates the $\mathrm{pH}$ of the solution. The ordinate on the left indicates the absorbance at $280 \mathrm{~nm}$ (mAU: milliabsorbance units). The abscissa indicates the elution volume. The solid and dashed lines show the elution patterns of IgG on affinity columns with immobilized PAB variants as shown in the Figure and the $\mathrm{pH}$ value of the elution buffer, respectively.

Additional file 6: Table S3. $P A B$ variants data by mutational experiments $[7,11,21-24]$ and by molecular simulation calculations [25-26]. The binding affinity of IgG for PAB variants was determined using ELISA and SPR. None: Almost the same binding affinity, Very small: 2-fold to < 5-fold decrease, Small: 5-fold to < 10-fold decrease, Large: 10-fold to $<$ 100-fold decrease, Very large: 100-fold decrease <.

Additional file 7: Figure S5. Circular dichroism melting curves of $P A B$ variants. (a) The curves of the single histidine substituted PAB variants. (b) The curves of the double histidine substituted PAB variants. Circular dichroism melting curves were obtained by monitoring the ellipticity at $222 \mathrm{~nm}$ with increasing temperature on a J-805 spectropolarimeter (JASCO). The mole fractions of the proteins in an unfolded state (thick lines) are shown as a function of temperature. Theoretical curves (thin lines) were calculated using a two-state equilibrium transition model.

Additional file 8: Figure S6. Binding efficiency of $P A B$ variants. The PAB variant (about $72 \mu \mathrm{g}$ ) was immobilized on NHS-activated agarose gel. The IgG (about 1mg) solution was added to the PAB variant immobilized agarose gel, and the mixture was shaken at $25^{\circ} \mathrm{C}$ for $30 \mathrm{~min}$ in $\mathrm{pH} 9.0$ buffer (25 mM Tris- $\mathrm{HCl}(\mathrm{pH}=9.0), 2.5 \mathrm{M} \mathrm{NaCl}$, and 0.1\% Tween 20). The amounts of IgG in supernatant after centrifugation were determined and the binding efficiency was calculated. The ordinate indicates the binding efficiency.

Additional file 9: Table S4. Amino acid residues of therapeutic antibodies related to the effects of $\mathrm{D} 36 \mathrm{H}$. In this study, Arg2519 of IgG-Fab was found to be related to the effects of D36H mutation. Of top 10 therapeutic antibodies in $2010,80 \%$ of the amino acid residues corresponding to Arg2519 are positively charged residues. Sales rank of therapeutic antibodies in 2010 are cited from the article (John G. Elvin et al. International Journal of Pharmaceutics 440 (2013) 83-98).

Additional file 10: Figure S7. Elution profile of lgG on affinity columns with immobilized 4XPAZ variants. (a) Elution profile of IgG from affinity columns prepared with immobilized $4 \times P A Z 01$ or $4 \times P A Z 03$. IgG was bound with $\mathrm{pH} 7.5$ buffer ( $25 \mathrm{mM}$ Tris- $\mathrm{HCl}$ ( $\mathrm{pH}=7.5$ ), $150 \mathrm{mM} \mathrm{NaCl}$, and $0.1 \%$ Tween 20 ) at a flow rate of $0.5 \mathrm{~mL} / \mathrm{min}$, (b) Elution profile of $\mathrm{lgG}$ from affinity columns prepared with immobilized 4XPAZ01 or 4XPAZ03. IgG was bound with pH 7.5 buffer ( $25 \mathrm{mM}$ Tris- $\mathrm{HCl}(\mathrm{pH}=7.5), 150 \mathrm{mM}$ $\mathrm{NaCl}$, and $0.1 \%$ Tween 20 ) at a flow rate of $0.1 \mathrm{~mL} / \mathrm{min}$, (c) Elution profile of IgG from affinity columns prepared with immobilized $4 \times$ PAZ01 or 4XPAZ03. IgG was bound with $\mathrm{pH} 9.0$ buffer (25 mM Tris- $\mathrm{HCl}$ ( $\mathrm{pH}=9.0)$, $2.5 \mathrm{M} \mathrm{NaCl}$, and $0.1 \%$ Tween 20 ) at a flow rate of $0.1 \mathrm{~mL} / \mathrm{min}$. Affinity columns were prepared using the $4 \times P A Z$ variants. The captured IgG on the column was eluted using a buffer with the $\mathrm{pH}$ indicated in the Figure. Afterwards, the residual lgG was eluted with a decreasing $\mathrm{pH}$ gradient. The ordinate on the right indicates the $\mathrm{pH}$ of the solution. The ordinate on the left indicates the absorbance at $280 \mathrm{~nm}$ (mAU: milliabsorbance units). The abscissa indicates the elution volume. The solid and dashed lines show the elution patterns of IgG on 4XPAZ variant columns, as shown in the Figure, and the $\mathrm{pH}$ value of the elution buffer, respectively. The inlets show the elution patterns with magnified scale from 0 to $8 \mathrm{~mL}$.

Additional file 11: Table S1. List of primers. Primers 1 4 indicate the oligonucleotides for constructing a histidine-scanning library. Primers 5 12 indicate the oligonucleotides for introducing histidine-mutations. The following abbreviations are used for mixed bases: $R=(A$ or $G)$, $Y=(C$ or $T), M=(A$ or $C), K=(G$ or $T), S=(G$ or $C)$ and $W=(A$ or $T)$.

\section{Abbreviations}

SpA: Staphylococcal protein A; Ig: Immunoglobulin; PAB: Staphylococcal protein A B-domain; PAZ: Staphylococcal protein A Z-domain. 


\section{Competing interests}

We declare that all authors are inventors on a pending patent using these Staphylococcal protein A variants.

\section{Authors' contributions}

MT conducted experiments, analyzed data, and co-wrote the manuscript $\mathrm{HW}$ and $\mathrm{AO}$ conducted experiments and analyzed data. SH designed the study, analyzed data and co-wrote the manuscript. All authors read and approved the final manuscript.

\section{Acknowledgements}

We thank Dr. Takashi Shimizu for critical reading of the manuscript. This study was partly supported by a grant for the project to build infrastructure for creating next-generation drugs for personalized medicine from the Ministry of Economy, Trade and Industry, Japan.

\section{Author details}

'Department of Medical Genome Sciences, Graduate School of Frontier Sciences, The University of Tokyo, Kashiwa, Japan. ${ }^{2}$ Biomedical Research Institute National Institute of Advanced Industrial Science and Technology (AIST), Higashi, Tsukuba, Ibaraki 305-8566, Japan. ${ }^{3}$ Manufacturing Technology Association of Biologics, Chuo-ku, Kobe, Japan.

Received: 1 March 2014 Accepted: 21 June 2014

Published: 1 July 2014

\section{References}

1. Brekke O, Sandlie I: Therapeutic antibodies for human diseases at the dawn of the twenty-first century. Nat Rev Drug Discov 2003, 2:52-62.

2. Maggon K: Monoclonal antibody "gold rush". Curr Med Chem 2007, 14:1978-1987.

3. Shukla A, Hubbard B, Tressel T, Guhan S, Low D: Downstream processing of monoclonal antibodies-application of platform approaches. J Chromatogr B 2007, 848:28-39.

4. Hermeling S, Crommelin D, Schellekens H, Jiskoot W: Structure-immunogenicity relationships of therapeutic proteins. Pharm Res 2004, 21:897-903.

5. Schellekens H: How to predict and prevent the immunogenicity of therapeutic proteins. Biotechnol Annu Rev 2008, 14:191-202.

6. Li R, Dowd V, Stewart D, Burton S, Lowe C: Design, synthesis, and application of aprotein A mimetic. Nat Biotechnol 1998, 16:190-195.

7. Brown N, Bottomley S, Scawen M, Gore M: A study of the interactions between an IgG-binding domain based on the $B$ domain of staphylococcal protein A and rabbit lgG. Mol Biotechnol 1998, 10:9-16.

8. Gulich S, Uhlen M, Hober S: Protein engineering of an IgG-binding domain allows milder elution conditions during affinity chromatography. J Biotechnol 2000, 76:233-243.

9. Watanabe $H$, Matsumaru $H$, Ooishi A, Feng $Y$, Odahara $T$, Suto $K$, Honda S: Optimizing $\mathrm{pH}$ response of affinity between protein $\mathrm{G}$ and IgG Fc. J Biol Chem 2009, 284:12373-12383.

10. Gera N, Hill A, White D, Carbonell R, Rao B: Design of pH Sensitive Binding Proteins from the Hyperthermophilic Sso7d Scaffold. PLoS One 2012, 7(11):e48928.

11. Watanabe H, Matsumaru H, Ooishi A, Honda S: Structure-based histidine substitution for optimizing $\mathrm{pH}$-sensitive Staphylococcus protein A. J Chromatogr B 2013, 929:155-160.

12. Tawfik D, Chap R, Eshhar Z, Green B: pH on-off switching of antibody-hapten binding by site-specific chemical modification of tyrosine. Protein Eng 1994, 7:431-434.

13. Morag E, Bayer E, Wilchek M: Reversibility of biotin-binding by selective modification of tyrosine in avidin. Biochem J 1996, 316:193-199.

14. Linder M, Nevanen T, Teeri TT: Design of a pH-dependent cellulosebinding domain. FEBS Lett 1999, 447:13-16.

15. Sarkar C, Lowenhaupt K, Horan T, Boone T, Tidor B, Lauffenburger D: Rational cytokine design for increased lifetime and enhanced potency using pH-activated "histidine switching". Nat Biotechnol 2002, 20:908-913.

16. Marttila A, Hytonen V, Latinen O, Bayer E, Wilchek M, Kulomaa M: Mutation of the important Tyr-33 residue of chicken avidin: functional and structural consequences. Biochem J 2003, 369:249-254.

17. Nordlund HR, Hytonen VP, Laitinen OH, Uotila ST, Niskanen EA, Savolainen J, Porkka E, Kulomaa MS: Introduction of histidine residues into avidin subunit interfaces allows $\mathrm{pH}$-dependent regulation of quaternary structure and biotin binding. FEBS Lett 2003, 555:449-454.

18. Igawa T, Ishii S, Tachibana T, Maeda A, Higuchi Y, Shimaoka S, Moriyama C, Watanabe T, Takubo R, Doi Y, Wakabayashi T, Hayasaka A, Kadono S, Miyazaki T, Haraya K, Sekimori Y, Kojima T, Nabuchi Y, Aso Y, Kawabe Y, Hattori K: Antibody recycling by engineered $\mathrm{pH}$-dependent antigen binding improves the duration of antigen neutralization. Nat Biotechnol 2010, 28:1203-1207.

19. Murtaugh M, Fanning S, Sharma T, Terry A, Horn J: A combinatorial histidine scanning library approach to engineer highly $\mathrm{pH}$-dependent protein switches. Protein Sci 2011, 20:1619-1631.

20. Deisenhofer J: Crystallographic Refinement and Atomic Models of a Human Fc Fragment and Its Complex with Fragment B of Protein A from Staphylococcus aureus at 2.9- and 2.8-A Resolution. Biochemistry 1981, 20:2361-2396.

21. Gore M, Ferris W, Popplewell A, Scawen M, Atkinson T: pH-sensitive interactions between IgG and a mutated IgG-binding protein based upon two B domains of Protein A from Staphylococcus aureus. Protein Eng 1992, 5:577-582.

22. Cedergren L, Andersson R, Jansson B, Uhlen M, Nilsson B: Mutational analysis of the interaction between staphylococcal protein $A$ and human IgG1. Protein Eng 1993, 6:441-448.

23. Tashiro M, Montelione G: Structures of bacterial immunoglobulin-binding domains and their complexes with immunoglobulins. Curr Opin Struct Biol 1995, 5:471-481.

24. Jendeberg L, Persson B, Andersson R, Karlsson R, Uhlen M, Nilsson B: Kinetic analysis of the interaction between protein $A$ domain variants and human Fc using plasmon resonance detection. J Mol Recognit 1995, 9:270-278.

25. Salvalaglio M, Zamolo L, Busini V, Moscatelli D, Cavallotti C: Molecular modeling of protein A affinity chromatography. J Chromatogr A 2009, 1216:8678-8686.

26. Huang B, Liu F, Dong $X$, Sun Y: Molecular mechanism of the affinity interactions between protein $A$ and human immunoglobulin $\mathrm{G} 1$ revealed by molecular simulations. J Phys Chem B 2011, 115(14):4168-4176.

27. Hillson J, Karr N, Oppliger I, Mannik M, Sasso E: The structural basis of germline-encoded VH3 immunoglobulin binding to staphylococcal protein A. J Exp Med 1993, 178:331-336.

28. Jansson B, Uhlen M, Nygren P: All individual domains of staphylococcal protein A show Fab binding. FEMS Immunol Med Microbiol 1998, 20:69-78.

29. Starovasnik M, O'connell M, Fairbrother W: and F. Kelley, R.: Antibody variable region binding by Staphylococcal protein A: thermodynamic analysis and location of the Fv binding site on E-domain. Protein Sci 1998, 8:1423-1431.

30. Graille M, Stura E, Corper A, Sutton B, Taussig M, Charbonnier J, Silverman GJ: Crystal structure of a Staphylococcus aureus protein A domain complexed with the Fab fragment of a human IgM antibody: Structural basis for recognition of B-cell receptors and superantigen activity. Proc Natl Acad SC US A 2000, 97:5399.

31. Winn MD, Ballard CC, Cowtan KD, Dodson EJ, Emsley P, Evans PR, Keegan RM, Krissinel EB, Leslie AGW, McCoy A, McNicholas SJ, Murshudov GN, Pannu NS, Potterton EA, Powell HR, Read RJ, Vagin A, Wilson KS: Overview of the CCP4 suite and current developments. Acta Crystallogr D Biol Crystallogr 2011, 67:235-242

32. Tsodikov O, Record MT Jr, Sergeev TV: Novel computer program for fast exact calculation of accessible and molecular surface areas and average surface curvature. J Comput Chem 2002, 23:600-609.

33. Honda S, Kobayashi N, Munekata E: Thermodynamics of a beta-hairpin structure: evidence for cooperative formation of folding nucleus. $\mathrm{J} \mathrm{Mol}$ Biol 2000, 295:269-278.

34. Makhatadze G, Privalov PL: Energetics of protein structure. Adv Protein Chem 1995, 47:307-417.

35. Honda S, Yamasaki K, Sawada Y, Morii H: 10 residue folded peptide designed by segment statistics. Structure 2004, 12:1507-1518.

doi:10.1186/1754-1611-8-15

Cite this article as: Tsukamoto et al.: Engineered protein A ligands, derived from a histidine-scanning library, facilitate the affinity purification of IgG under mild acidic conditions. Journal of Biological Engineering 2014 8:15. 